\title{
CAPILLARY ARRAY ELECTROPHORESIS BIOPROCESSORS
}

\author{
Richard A. Mathies, Eric T. Lagally, Toshihiro Kamei, William H. Grover, \\ Chung N. Liu and James R. Scherer \\ Department of Chemistry, University of California, Berkeley, CA 94720 \\ Robert A. Street \\ Palo Alto Research Center, Palo Alto, CA 94314
}

\begin{abstract}
Microfabricated capillary array electrophoresis devices have been developed that provide unprecedented analysis speed and throughput. The next challenge is the development of integrated arrays of valves, vents, pumps and reactors to provide nanoliter volume sample preparation and the development of integrated detectors to facilitate portable devices. This paper presents our current work on the development of integrated pneumatic valves and pumps that operate on the nanoliter scale, microfabricated resistive heaters and temperature sensors for integrated sample preparation, and integrated hydrogenated amorphous $\mathrm{Si}$ photodiode detectors that enable point-of-analysis devices.
\end{abstract}

\section{INTRODUCTION}

MEMS technologies are now being applied with great success to the development of microfabricated "lab-on-achip" chemical and biochemical analysis devices. The earliest successful devices consisting of single channels etched in silica substrates were used to perform rapid capillary electrophoretic (CE) analyses of fluorescent dyes and amino acids [1]. This work rapidly spawned the application of microfabricated CE devices to DNA fragment sizing [2], DNA sequencing [3, 4], protein analyses [5], and clinical measurements [6]. Virtually all classes of chemical and biochemical analyses can be reduced to a chip-based format. However, the power of MEMS is not just its ability to make a compact device for rapid analyses. Microfabrication importantly and uniquely enables the production of dense arrays of analysis devices providing high throughput and low analysis cost.

The development of arrays of chemical and biochemical analysis systems integrated into wafer-scale devices presents unique opportunities and challenges. Our early work presented rectilinear arrays of 12 and 48 separation channels that were detected by mechanical and galvoscanning confocal fluorescence $[7,8]$. However, we found that these formats were not optimal because the devices were hard to layout, they contained deleterious turns, extension to larger channel numbers was not facile, and each new chip layout required a new scanner configuration. The transition to radial channel layouts effectively addressed all of these limitations. Radially arrayed channels are easily designed in groups of from 2 to 4 separation channel-injector modules that are replicated by exploiting the radial symmetry. Detection is accomplished with the Berkeley rotary confocal fluorescence scanner that is easily adapted to wafers of different diameters, and can detect from one to 384 channels in its current configuration.

A typical microfabricated capillary array electrophoresis $(\mu \mathrm{CAE})$ wafer layout is presented in Figure 1 along with an expanded view of one channel-injector module. 96 Channels are arrayed radially with the cathode end of the separation channels on the perimeter and the common anode at the center, providing a $5.5 \mathrm{~cm}$ separation distance on the $150-\mathrm{mm}$ diameter wafer. Two samples are introduced in adjacent sample reservoirs and then loaded through the cross channel injector by applying electrophoretic potential between the sample reservoirs and the common waste reservoir. Once the intersection is charged with analyte, the analyte is separated by applying a potential from the common cathode to the central anode, driving the analyte through a sieving gel for size-based separation.

The analyte is detected as it migrates to the anode by the rotary confocal fluorescence scanner depicted in Figure 1C. Light at $488 \mathrm{~nm}$ is introduced into the optical system at a dichroic beamsplitter and passed up through a rotating rhomb prism and microscope objective so that the focused exciting light scans in a $1-\mathrm{cm}$ radius circle about the central anode. The fluorescence emitted when the labeled analyte passes this "finish line" is gathered by the objective, descanned by the rhomb prism, and passed to a four-color confocal fluorescence detector. The scanner operates at rates from a few to $20 \mathrm{~Hz}$, and it collects four 16-bit data points every 20 microns about the $6.28 \mathrm{~cm}$ circumference detection circle. The detection sensitivity of this scanner is typically in the picomolar range.

This chip and detection format have now been used for a wide variety of biochemical analyses. Using noncovalent intercalation dyes for 2-color labeling, Shi et al. analyzed 96 human genomic samples for genetic variations in the methylenetetrahydrofolate reductase gene in only $120 \mathrm{~s}$ [9]. Medintz et al. performed human identity determination on 96 genomic samples by analyzing multiplexed short tandem repeats in under 8 minutes [10]. Paegel et al. recently presented the development of a novel folded channel DNA sequencing microchannel plate and demonstrated its utility by performing DNA sequencing to 500 bases per lane on a 96lane device [11]. Finally, recent work by Emrich et al. presented the fabrication of a 384-lane $\mu \mathrm{CAE}$ device on a $200-\mathrm{mm}$ diameter wafer and demonstrated its utility by typing human genomic samples for a common genetic variation associated with hereditary haemochromatosis [12]. Figure 2 summarizes progress in the development and application of $\mu \mathrm{CAE}$ systems. Our first analyses on single 
channels detected a single sample in $120 \mathrm{~s}$ [2]. The throughput has rapidly climbed over the years. Our most recent results using $200 \mathrm{~mm}$ dia. wafers with 384 separation channels produced throughputs of 2 samples per second of operation - an increase of nearly 300 -fold in only 8 years.

The dramatic advances in analytical capabilities of microfabricated electrophoretic analysis systems along with the equally dramatic advances in the number of analyses that can be performed on a given wafer, present important new challenges for the field. While the promise of large numbers of high-speed analyses has been dramatically met by
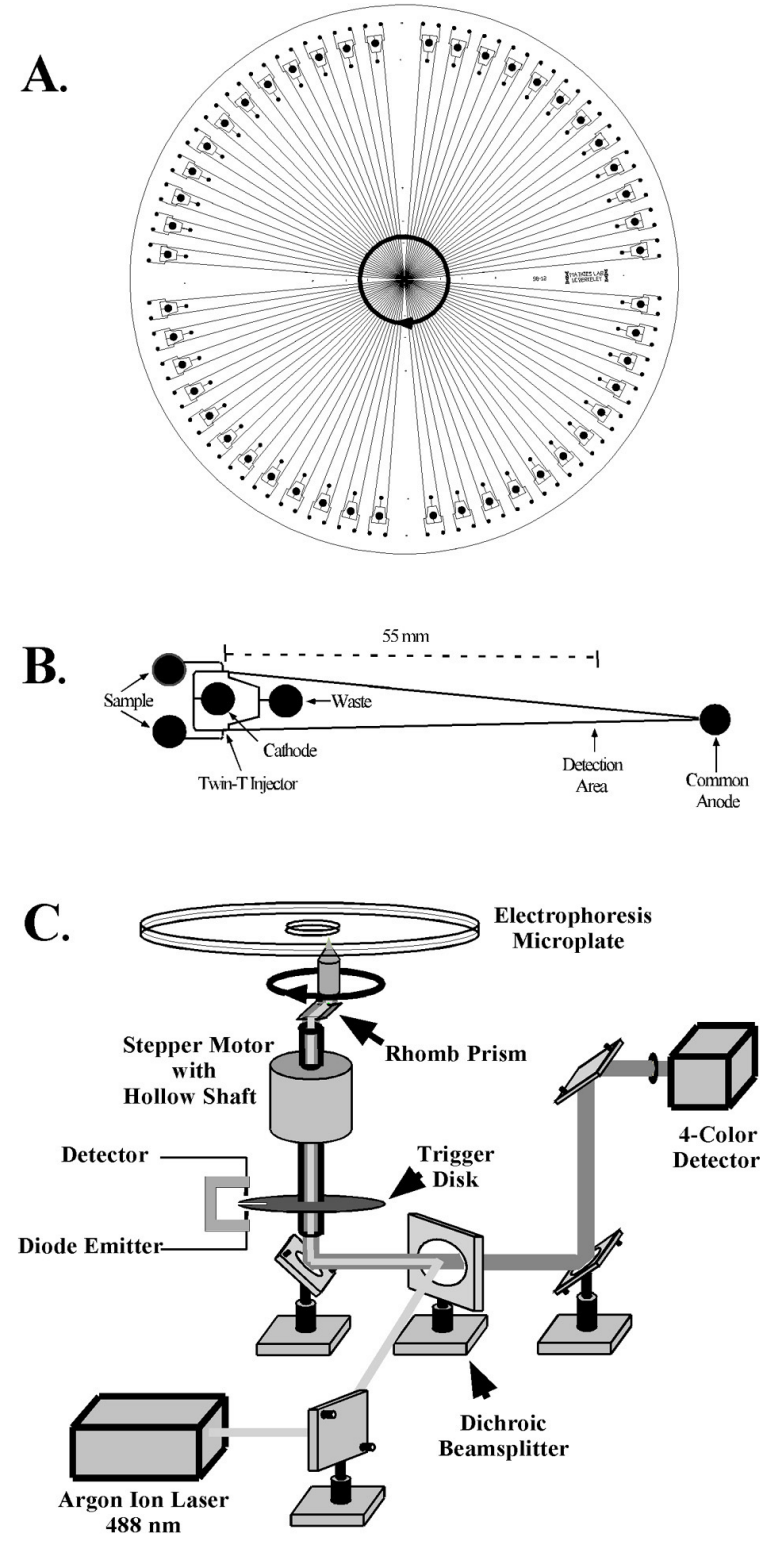

Figure 1. (A) Design of a 96-channel microfabricated radial capillary array electrophoresis microchannel plate. (B) Expanded view of a pair of separation channels showing the common cathode and waste reservoirs and the twin- $T$ injector. (C) Design of the Berkeley confocal fluorescence rotary scanner. Adapted from Shi et al. [9].

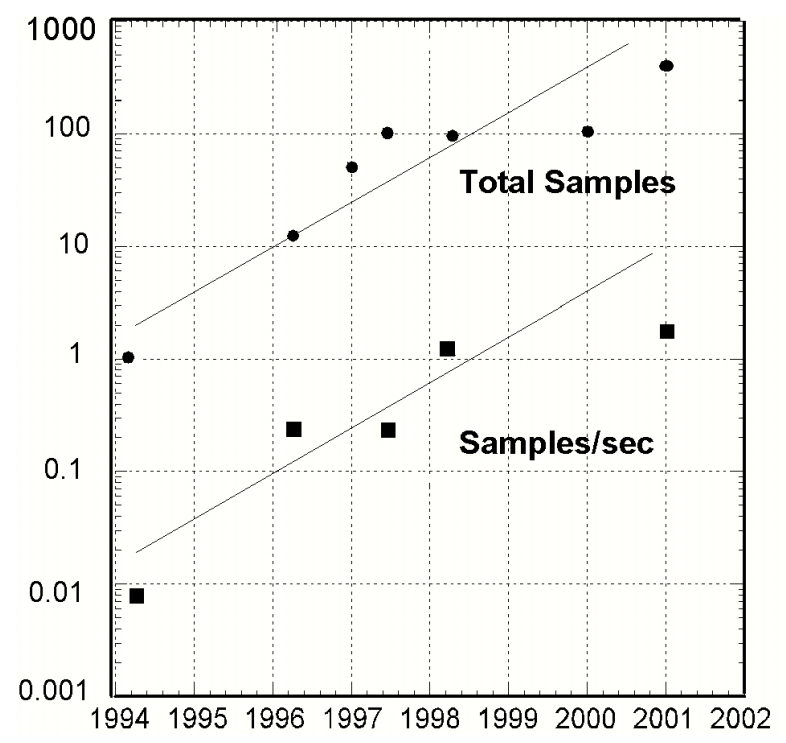

Figure 2. Performance advances of microfabricated capillary array electrophoresis devices over the past 8 years.

$\mu \mathrm{CAE}$ systems, the promise to reduce sample volumes and hence costs from microliters to nanoliters has not been delivered. The use of microfluidic channels provides in principle the ability to transport nanoliter volumes, to react nanoliter volumes, to clean-up and concentrate these small volumes, and load them on analysis channels. No one has yet presented a convincing general paradigm for accomplishing this important goal. Achieving this goal is critical if we are to have a facile, low-cost, and rapid way to introduce samples into the high-throughput analyzers described above. The purpose of this paper is to present current work in our lab directed toward the development of fully integrated microdevices for manipulating, reacting and detecting chemical and biochemical analytes.

\section{INTEGRATED VALVE STRUCTURES}

A key challenge in the development of integrated nanoliter sample preparation is the facile production of low dead-volume valves. The utility of pneumatically driven membrane flex valves for $\mu \mathrm{L}$ fluidics [13] encouraged us to develop a nanoliter version of this valve. Figure 3 presents the fluidic characteristics of the latex membrane valves that we have developed [14]. Figure 3A plots the external pressure required to force sample through a valve closed with the indicated valve pressure. The valves are capable of withstanding only a small forward pressure of $\leq 20 \mathrm{kPa}$. The breakdown pressure, defined as the inflection point in Figure $3 \mathrm{~A}$, occurs at approximately $10 \mathrm{kPa}$. Figure 3B presents the flow rate of water through the valve at different valve positions. All flow rates were determined using a $14 \mathrm{kPa}$ forward pressure. The flow rate rapidly surpasses $300 \mathrm{~nL} / \mathrm{s}$ at very small membrane deflections; the maximum flow rate is $360 \mathrm{~nL} / \mathrm{s}$. Despite the simple 
fabrication procedure, these valves have demonstrated their utility through the performance of $\mathrm{nL}$ PCR coupled to $\mathrm{CE}$ analysis on a chip $[\mathbf{1 5}, \mathbf{1 6}]$.

While these discrete pneumatic valves have worked well, their fabrication is problematic for high density devices. Thus, we desired a more routine and transferable fabrication method. We therefore developed a PDMS membrane valve composed of four components: a channel layer, a fluidic port layer, a polydimethylsiloxane (PDMS) membrane and a drilled access layer. Figure 4 presents a cross-sectional schematic of the PDMS membrane valve.
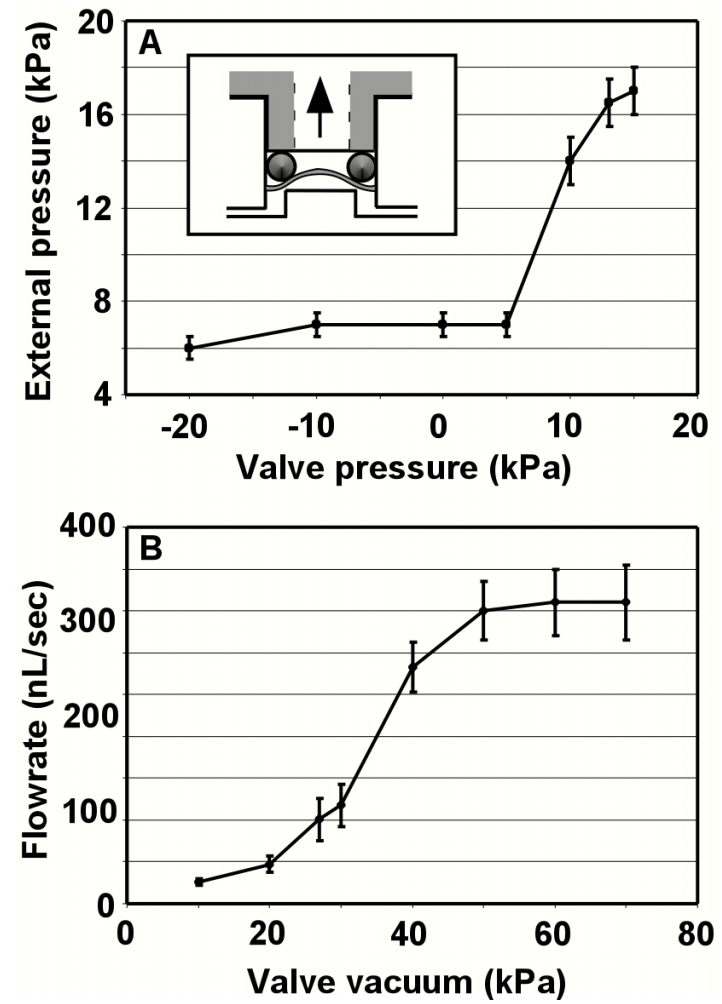

Figure 3. (A) Breakdown pressure of the latex membrane valve. (B) Flow rate of water through the latex membrane valve as a function of the activation vacuum with a pressure head of $14 \mathrm{kPa}$. The inset presents the design of the latex membrane valve.

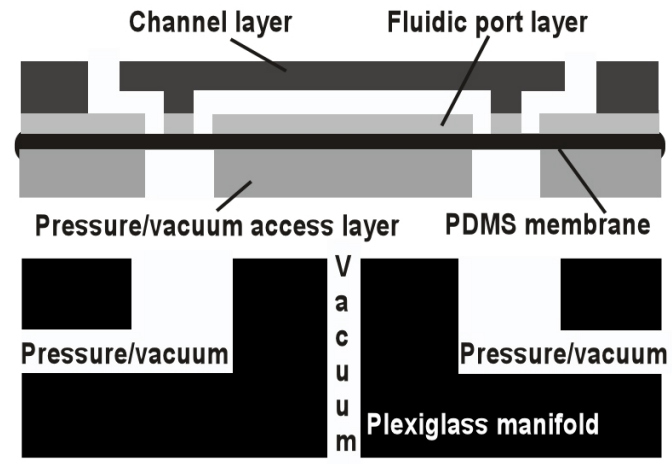

Figure 4. Schematic of the PDMS membrane valve with plexiglass manifold.
The channel layer is formed by bonding a 550- with a 210- $\mu \mathrm{m} 10-\mathrm{cm}$ diameter glass wafer. 0.01" Diameter holes were drilled in the $210-\mu \mathrm{m}$ glass wafer to act as fluidic ports for the valve. By using a thin $210-\mu \mathrm{m}$ glass wafer and drilled holes of $254-\mu \mathrm{m}$ in diameter, valves with extremely small dead volumes $(\sim 20 \mathrm{~nL})$ can be produced. The pressure/vacuum access layer was made of a $1.1-\mathrm{mm}$, $10 \mathrm{~cm}$ diameter glass wafer. $1.5-\mathrm{mm}$ holes were drilled on the wafer to deliver vacuum/pressure from an external Plexiglass manifold. The PDMS membrane valve is formed by sandwiching a 0.007" thick PDMS membrane between the channel layer and the access layer. Reproducible and leak-free bonding was obtained with this method.

The performance of the PDMS membrane valve was characterized by determining the breakdown pressure of the valve, the flow rate of water across the valve, and membrane durability testing. The breakdown pressure of valve is defined as the minimum external pressure needed to force liquid to leak through the valve when the valve is set under various pressure and vacuum levels. Figure 5 presents the valve breakdown pressure of the PDMS membrane valves. The breakdown pressure is as high as $\sim 75 \mathrm{kPa}$ with an applied valve pressure of $40 \mathrm{kPa}$. It is very important to have such a high breakdown pressure to ensure a reliable and leak-free valve.

The flow rate of water through the valve was determined by measuring the time it takes to fill a channel of known volume. Figure $5 \mathrm{~B}$ presents the flow rate
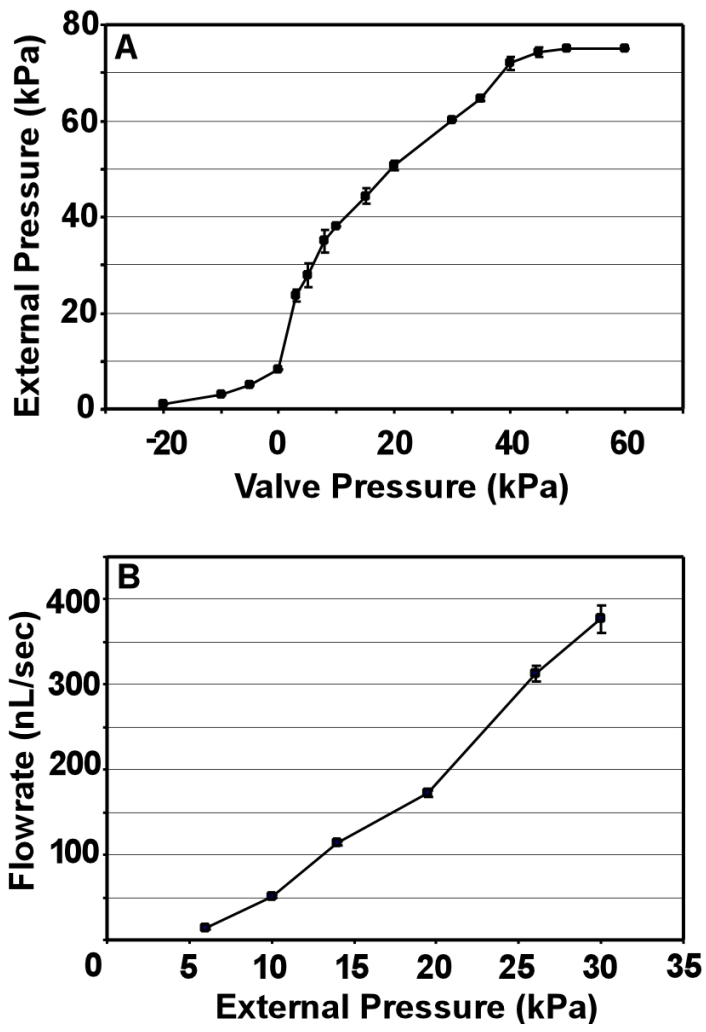

Figure 5. (A) Valve breakdown pressure vs. applied pressure for the PDMS membrane valve. (B) Flow rate of water vs. applied pressure for the PDMS membrane valve. 


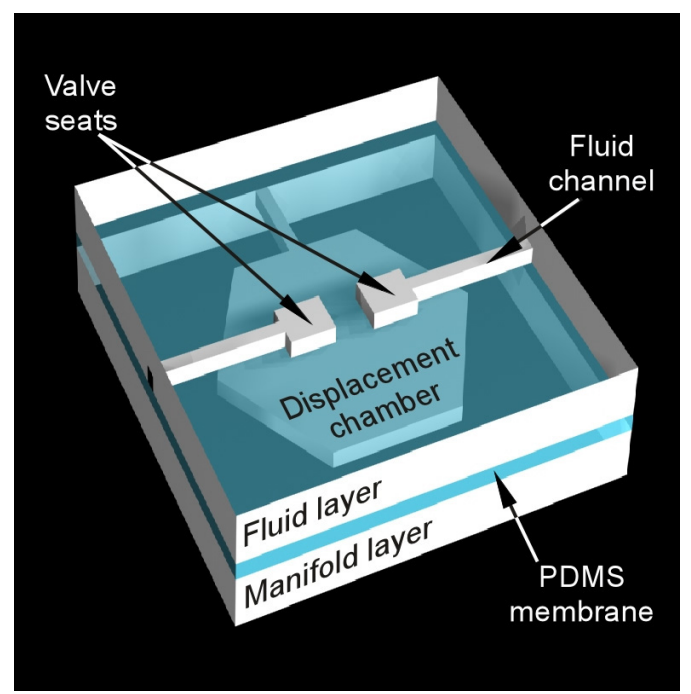

Figure 6. CAD view of an integrated valve.
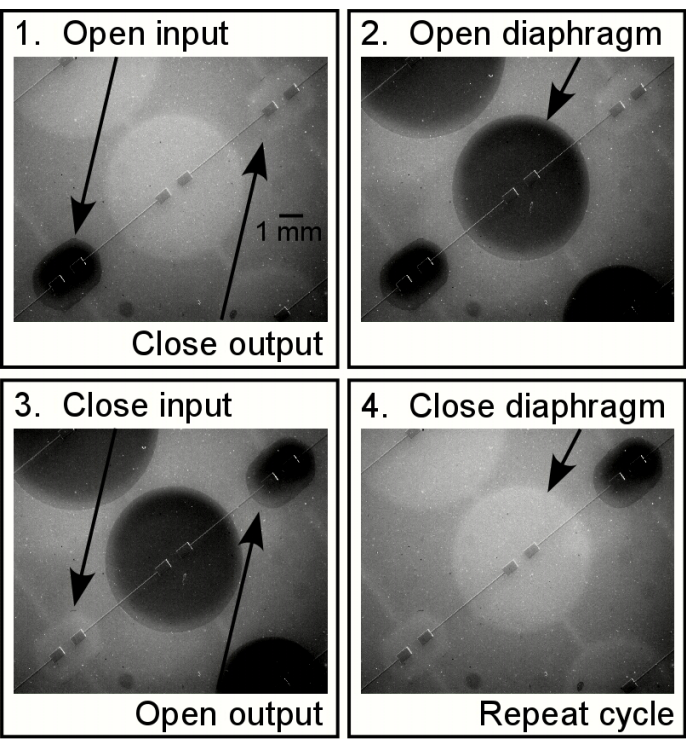

Figure 7. Actuation of a microfabricated PDMS diaphragm pump.

through the PDMS valve as a function of applied pressure. The flow rate was linear in the applied pressure, and flow rates as high as $\sim 380 \mathrm{~nL} / \mathrm{s}$ are achieved by applying an external pressure of $30 \mathrm{kPa}$. No significant changes of deflection have been observed with more than 100 actuations. This demonstrates that the PDMS membrane valve is very durable and reliable for long-term use.

\section{VALVE ARRAYS AND PUMPS}

We have also developed methods to make arrays of integrated valves. In this design, pneumatic connections are integrated into the device, allowing many more valves to be operated in parallel. The three-layer design of the integrated valve is shown in Figure 6. The manifold layer contains etched channels that distribute vacuum or pressure to valves in the device; the etched channels on the fluidic layer contain the fluid to be routed or pumped. The threelayer topology of integrated valves allows several valves to be controlled by the same vacuum/pressure line, thereby maximizing the number of valves on the device while minimizing the number of pneumatic connections. In an integrated valve, the membrane deflects away from the fluid layer and into an etched displacement chamber in the manifold layer. The volume of the displacement chamber determines the volume of fluid that can be drawn into the valve.

We have demonstrated that a novel microfluidic diaphragm pump can be constructed by placing three integrated valves in series. The pumps are operated by actuating the constituent valves according to the cycle shown in Figure 7. Reversing the actuation cycle reverses the direction of fluid pumping. All 48 pumps are controlled by only nine pneumatic connections placed at the edge of the device. The volume pumped per actuation was determined by counting the actuations required to pump a known volume of solution; pumps were found to range from $94 \mathrm{~nL}$ to $7.1 \mu \mathrm{L}$ per actuation depending on the displacement chamber volume. A series of five circularshaped pumps were used to quantify the relationship between diaphragm valve size and volume pumped per actuation. These results suggest that a circular diaphragm pump will pump approximately $72 \%$ of the volume of the displacement chamber of its diaphragm valve, and that the relationship between the volume pumped and the displacement chamber volume is linear. This work demonstrates that diaphragm pumps may be designed to accurately and reproducibly pump specific volumes in applications where nanoliter to microliter-scale control of fluids is required.

\section{INTEGRATED HEATERS AND TEMPERATURE SENSORS}

We have also developed fully integrated heaters and Pt resistance temperature detectors (RTDs) for our genomic analysis microdevices. The heaters are fabricated on the backside of the device, and consist of parallel sets of $\mathrm{Ti} / \mathrm{Pt}$ resistors with gold leads to the edge of the device for electrical contact. The leads are electroplated gold to decrease their resistance, which allows for localized heating at the chamber of interest. The Ti/Pt RTDs are fabricated within the glass microreactors, and are of a fourwire design, which minimizes noise and systematic error due to self-heating effects. Figure 8A presents a scanning electron micrograph of a completed RTD. Electrical current is applied through the two outside leads, and voltage as a function of temperature is sensed on the inside leads. The dependence of voltage on resistance is expected to be linear for a Pt RTD over our narrow temperature range. Figure $8 \mathrm{~B}$ presents a typical calibration curve for our Ti/Pt RTDs. The figure indicates a nearly perfectly linear dependence of measured voltage on applied current, although the actual fitted function is the Calendar Van Dusen Equation, which for temperatures above $0^{\circ} \mathrm{C}$ assumes a quadratic form: 

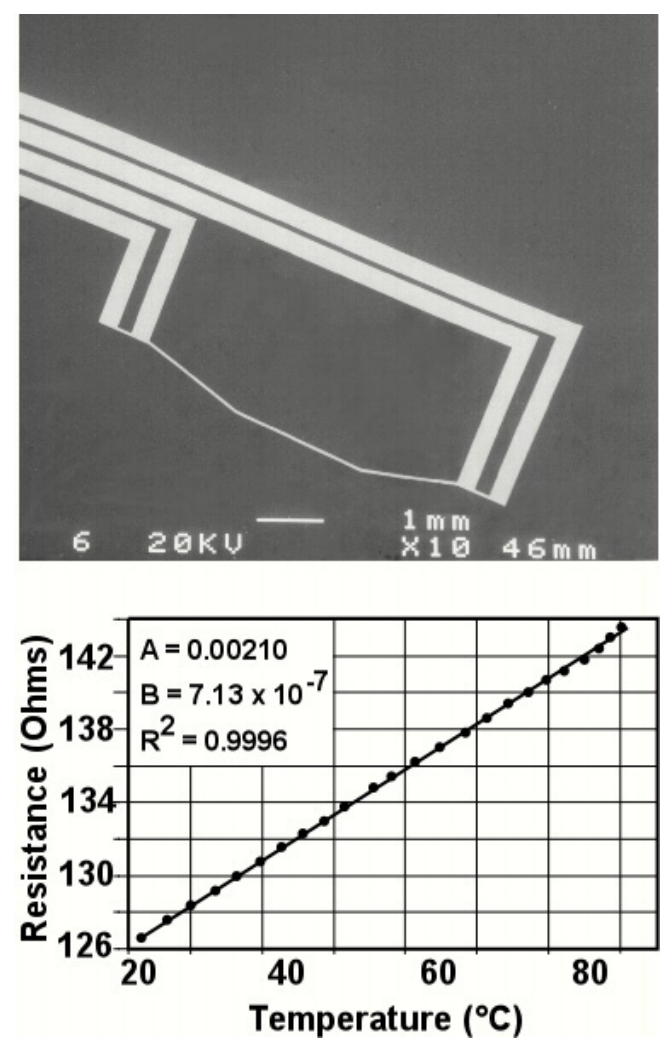

Figure 8. (A) Scanning electron micrograph of an integrated Ti/Pt RTD used for sensing temperature within microfabricated reactors. (B) A typical calibration curve for our RTDs.

$$
R_{1}=R_{0}\left(1+A T+B T^{2}\right)
$$

where $R_{t}$ is the resistance at temperature $T$ and $R_{0}$ is the RTD resistance at $0^{\circ} \mathrm{C}$.

\section{INTEGRATED DETECTORS}

The miniaturization and on-chip integration of the fluorescence detection system is a fundamental challenge that will enable the development of portable point-of-analysis devices. Conventional crystalline Si photodiodes have been proposed as integrated detectors [17]. Our work has been directed toward the use of hydrogenated amorphous silicon (a-Si:H) PIN photodiodes to fashion an integrated fluorescence detector. High sensitivity a-Si:H photodiodes can be fabricated by plasma enhanced chemical vapor deposition at a very low temperature of $\sim 200^{\circ} \mathrm{C}$. This permits the direct fabrication of a-Si:H photodiodes on inexpensive substrates such as glass or plastics. Moreover, many advantages of a-Si:H in manufacturing, patterning, and low production cost will be important in making arrays of photodiodes $[\mathbf{1 8}]$ and in making disposable devices.

Figure 9 presents our design for an integrated a-Si:H photodiode detector and optical system for $\mu \mathrm{CAE}$ devices. The annular shape of the a-Si:H detector allows us to use vertical laser excitation (e.g.. from a VCSEL) to keep laser light from directly impinging on the optical filter and the

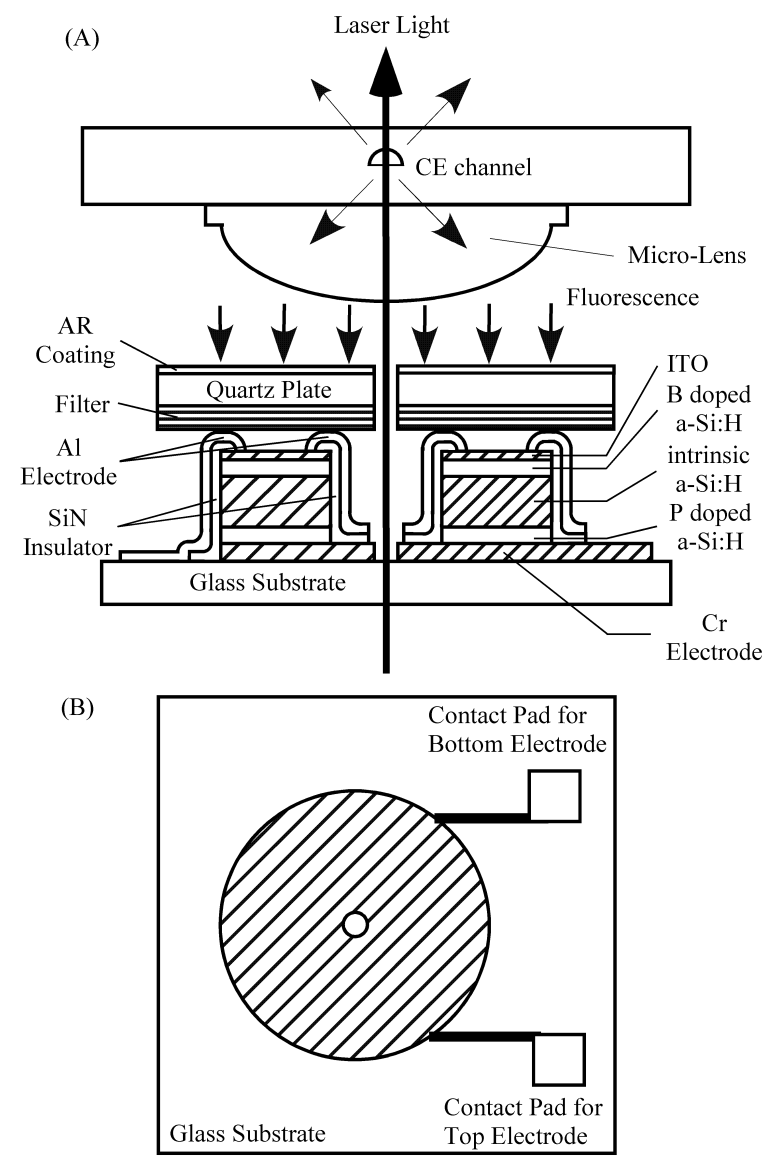

Figure 9. Design of an integrated a-Si:H photodiode and optical system for detecting analytes in microfabricated capillary electrophoresis channels. (A) Cross-sectional view. (B) Top view of annular a-Si:H photodiode detector.

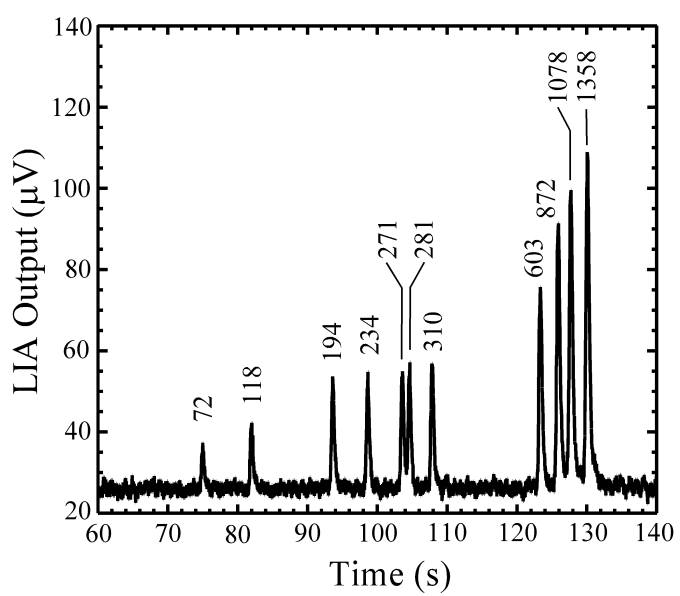

Figure 10. Electrophoretic separation of a $\phi X 174$ Hae III digest of DNA labeled with thiazole orange fluorescent dye and detected with an a-Si:H photodiode detector.

detector. First, laser light at $488 \mathrm{~nm}$ is introduced normal to the $\mu \mathrm{CAE}$ plate through pinholes in the detector and optical filter. The fluorophore in the channel is illuminated by the laser light, producing fluorescence. The fluorescence is collected by a micro-lens such as a half-ball lens or aspheric 
lens, and transmitted by a long-pass filter such as a multilayer $\mathrm{ZnS} / \mathrm{YF}_{3}$ interference filter that simultaneously eliminates the excitation light. Finally, fluorescence reaches the a-Si:H detector and a photocurrent is generated.

To explore the feasibility of this design, DNA fragment sizing was performed and detected with an a-Si:H detector. Fluorescence labeling of the $25 \mathrm{ng} / \mu \mathrm{l}$ sample of $\phi 174 \mathrm{HaeIII}$ DNA digest ladder was accomplished with $1 \mu \mathrm{M}$ of an intercalating dye (thiazole orange). The DNA samples were injected by the plug injection method and electrophoresis was carried out at $300 \mathrm{~V} / \mathrm{cm}$. A reverse bias voltage of $1 \mathrm{~V}$ was applied to the a-Si:H diode, and the photocurrent synchronized to chopped laser light (Ar ion laser, $488 \mathrm{~nm}, 7$ $\mathrm{mW}$ ) was detected by a lock-in amplifier. The chopping frequency was $27 \mathrm{~Hz}$, while the time constant was $100 \mathrm{~ms}$. Figure 10 shows that all 11 peaks of this DNA ladder are successfully observed with a good $\mathrm{S} / \mathrm{N}$ ratio.

Further integration of the laser and detector is possible. Because of the compatibility of a-Si:H diodes with glass, it is feasible to monolithically integrate the detector with these glass wafer electrophoretic devices. The combination of a blue-green VCSEL with this detector module should lead to a simple "contact lens" type of excitation and detection module for microfabricated $\mathrm{CE}$ chips. Therefore, a-Si:H photodiodes are promising for use as integrated detectors, because they are easy to manufacture and pattern, should dramatically reduce production costs, and should facilitate the development of portable point-of-analysis devices.

\section{SUMMARY AND CONCLUSIONS}

The development of fully integrated chemical and biochemical microprocessors will depend on the development of facile reliable integrated sample preparation technologies as well as detection methods. We have presented a variety of methods for producing pneumatic valve structures, characterized their operation, and shown that they can be used to make PCR reactors, valve arrays and pumps. The fabrication of integrated temperature sensors and heaters is also presented. When combined with the development of integrated a-Si:H photodiode detectors and chip VCSEL sources, it is evident that portable point-of-analysis lab-on-achip devices are now feasible.

\section{REFERENCES}

1. D. J. Harrison, K. Fluri, K. Seiler, Z. Fan, C. S. Effenhauser, and A. Manz, "Micromachining a miniaturized capillary electrophoresis-based chemical analysis system on a chip", Science, 261, 895-897 (1992).

2. A. T. Woolley and R. A. Mathies, "Ultra-high-speed DNA fragment separations using microfabricated capillary array electrophoresis chips", Proc. Natl. Acad. Sci. U.S.A., 91, 11348-11352 (1994).

3. S. Liu, Y. Shi, W. W. Ja, and R. A. Mathies, "Optimization of high-speed DNA sequencing on microfabricated capillary electrophoresis channels", Anal. Chem., 71, 566-573 (1999).

4. D. Schmalzing, N. Tsao, L. Koutny, D. Chisholm, A. Srivastava, A. Adourian, L. Linton, P. McEwan, P. Matsudaira, D. Ehrlich, "Toward real-world sequencing by microdevice electrophoresis", Genome Res., 9, 853 (1999).

5. C. Colyer, D. Shakuntala, D. Mangru, and D. J. Harrison, "Microchip-based capillary electrophoresis of human serum proteins", Journal of Chromatography A, 781, 271 (1997).

6. H. J. Tian, L. C. Brody, S. J. Fan, Z. L. Huang, and J. P. Landers, Clinical Chemistry, 47, 173-185 (2001).

7. A. T. Woolley, G. F. Sensabaugh, and R. A. Mathies, "High-speed DNA genotyping using microfabricated capillary array electrophoresis chips", Anal. Chem., 69, 2181 (1997).

8. P. C. Simpson, D. J. Roach, A. T. Woolley, T. Thorsen, R. Johnston, G. F. Sensabaugh, and R. A. Mathies, "Highthroughput genetic analysis using microfabricated 96-sample capillary array electrophoresis microplates", Proc. Natl. Acad. Sci. U.S.A., 95, 2256-2261 (1998).

9. Y. Shi, P. C. Simpson, J. R. Scherer, D. S. Wexler, C. Skibola, M. T. Smith, and R. A. Mathies, "Radial capillary array electrophoresis microplate and scanner for highperformance nucleic acid analysis", Anal. Chem., 71, 53545361 (1999).

10. I. Medintz, L. Berti, C. A. Emrich, J. Tom, J. R. Scherer, and R. A. Mathies, "Genotyping energy-transfer cassette labeled short tandem repeat amplicons with capillary electrophoresis microchannel plates", Clinical Chemistry, 47, 1614-1621 (2001).

11. B. M. Paegel, C. A. Emrich, G. J. Wedemayer, J. R. Scherer, and R. A. Mathies, "High-throughput DNA sequencing with a 96-lane capillary array electrophoresis bioprocessor", Proc. Natl. Acad. Sci. U.S.A., 99, 574-579 (2002).

12. C. A. Emrich, H. Tian, I. L. Medintz, and R. A. Mathies, "Microfabricated 384-lane capillary array electrophoresis bioanalyzer for ultra high-throughput genetic analysis", Nature Biotechnology, submitted (2002).

13. R. C. Anderson, X. Su, G. J. Bogdan, and J. Fenton, "A miniature integrated device for automated multistep genetic assays", Nucleic Acids Research, 28, e60 (2000).

14. E. T. Lagally, P. C. Simpson, and R. A. Mathies, "Monolithic integrated microfluidic DNA amplification and capillary electrophoresis analysis system", Sensors and Actuators B, 63, 138-146 (2000).

15. E. T. Lagally, I. Medintz, and R. A. Mathies, "Singlemolecule DNA amplification and analysis in an integrated microfluidic device", Anal. Chem., 73, 565-570 (2001).

16. E. T. Lagally, C. A. Emrich, and R. A. Mathies, "Fully integrated PCR-capillary electrophoresis microsystem for DNA analysis", Lab-on-a-Chip, 1, 102-107 (2001).

17. J. R. Webster, M. A. Burns, D. T. Burke, and C. H. Mastrangelo, "Monolithic capillary electrophoresis device with integrated fluorescence detector", Analytical Chemistry, $73,1622-1626$ (2001).

18. R. A. Street and L. E. Antonuk, "Amorphous silicon arrays develop a medical image", IEEE Circuits and Devices Magazine, 9 (4) July, 38-42 (1993). 